\title{
Transcending disciplines in architecture, structural and building services engineering: a new multidisciplinary educational approach
}

\author{
Sonja Oliveira ${ }^{1} \cdot$ Luke Olsen $^{1} \cdot$ Liora Malki-Epshtein $^{2} \cdot$ Dejan Mumovic $^{1}$. \\ Dina D'Ayala ${ }^{1,2}$
}

Accepted: 5 December 2020 / Published online: 4 January 2021

(C) The Author(s) 2021

\begin{abstract}
This paper reflects upon the mechanisms that enable development of curricular approaches to multidisciplinary architecture/engineering higher education. Building upon recent calls for integrated multidisciplinary building design practice, academics at UCL, industry partners and respective professional bodies embarked upon developing a new course that challenged disciplinary boundaries and defined the needs of a new design professional. Whilst there have been attempts internationally to better integrate architecture as well as engineering education, efforts have largely been focused on bolt-on solutions based on pre-existing education programmes. In addition, there has been little discussion (empirical or theoretical) on practical measures associated with developing multidisciplinary education in the built environment. Drawing on mixed data including documentary evidence, semi structured interviews and observations, the study begins to shed light on the approaches underpinning the development of a multidisciplinary built environment MEng course at UCL that integrates architecture, building services and civil engineering. The paper's contribution is threefold. First, the findings have implications for developing multidisciplinary built environment education curricula, through revealing key mechanisms including the need for shared attitudes and expectations. Second, the paper highlights the conditions that enable the negotiation of multidisciplinary curricula including institutional support, shared values and a collective need and willingness to explore new solutions. Third, the paper reflects upon the value of design studio learning as a critical integrative component to the delivery of multidisciplinary education in the built environment and STEM more widely.
\end{abstract}

Keywords Architecture $\cdot$ Design studio $\cdot$ Engineering $\cdot$ Education $\cdot$ Multidisciplinary $\cdot$ STEM

Luke Olsen

1.olsen@ucl.ac.uk

1 UCL Institute for Environmental Design and Engineering, The Bartlett, UCL Faculty of the Built Environment, London WC1H 0NN, UK

2 Department of Civil, Environmental and Geomatic Engineering, UCL, London WC1E 6BT, UK 


\section{Introduction}

It is widely recognized in policy, industry and academia that built environment education can benefit from multidisciplinary curricula (Graham 2018; Dym et al. 2005). The historically siloed discipline-based structure of many built environment curricula, such as building services/civil/structural/environmental engineering or architecture are not meeting current or future industry needs (Borrego and Newswander 2008; Sunthonkanokpong 2011). In the UK, recent reports from construction think tanks such as the EDGE as well as professional bodies and institutions including the RIBA and the Royal Academy of Engineering highlight the need for collaborative multidisciplinary innovative education as critical for UK built environment professionals (Jones and Gloster 2018; MacLaren et al. 2017; Morell 2015; MacLaren et al. 2017). Similarly, in an international context, a recent report in the USA, Educating engineers: Preparing 21st Century Leaders in the Context of New Modes of Learning (National Academy of Engineering 2013), also advocate preparing building designers who can work in creative, interdisciplinary environments. The need for innovative educational approaches is particularly relevant in the construction sector where complex cross disciplinary problems require collaboration and integrated solutions drawn from across multiple disciplines (Becerik-Gerber et al. 2011). Consequently, many high-profile engineering and architecture departments are actively developing multidisciplinary learning opportunities and integrated teaching environments, often with industry partners involved. These 'emerging leaders' are seen to begin to deliver "distinctive, student-centered curricular experiences with an integrated and unified educational approach" (Graham 2018). However, the challenge remains to deliver such integrated, student-centered multidisciplinary built environment education at scale to larger cohorts of students.

There are limited studies that focus on or account for educators' experiences in developing built environment multidisciplinary courses. However, the following sections offer helpful insights drawn out of research on approaches to creating multidisciplinary learning opportunities in existing architecture and engineering modules. Learning opportunities for integrating multidisciplinary content are largely discussed through the lens of sustainability and/or project-based teaching in either engineering or architecture courses. Studies that examine the development of multidisciplinary content in engineering modules tend to mostly reflect upon (1) approaches to integrating multidisciplinary learning within teaching sessions or (2) the multidisciplinary content design process. Research that discusses integration of multidisciplinary content into architecture modules including engineering content, usually focuses primarily on the effects of the learning highlighting the value to students in their understanding of different types of learning modes.

Whilst multidisciplinary education is increasingly called upon in the construction sector, there have been few studies that examine the educational, social and organisational approaches and experiences involved in developing multidisciplinary courses (Becerik-Gerber et al. 2011; Macdonald and Granroth 2013), with most research discussing bolt-on multidisciplinary learning experiences or opportunities within particular course modules. Also, most studies tend to be descriptive with little or no critique of the process and/or outcomes of the approaches involved in discussing and designing a multidisciplinary curriculum, particularly how a multidisciplinary approach is defined, arrived at and evaluated. In addition, use of the terms multidisciplinary or interdisciplinary are largely ill defined and not well understood (Morse et al. 2007). 


\section{Background}

Research in architecture and engineering education has analyzed approaches to achieving multidisciplinary curricula mostly through either incorporating sustainability or enabling design-studio and/or project-based learning delivered by multiple disciplines. More recently, there has also been a focus placed on design thinking and BIM as a vehicle for enabling opportunities for multidisciplinary learning. The below review draws from literature on both engineering and architecture pedagogy. Examining existing studies on these educational disciplines highlights a lack of examples where multi-directional learning is developed or achieved. Rather, the majority of studies prioritise the importance of embedding architectural practice into engineering education or engineering approaches in architecture education. There are also no studies to date that discuss the ways a course with more than two disciplines could be created and the mechanisms that enable a multidisciplinary course rather than a module.

Integrating sustainability and drawing upon multiple disciplinary views are often viewed as a way of achieving a multidisciplinary curriculum. Thürer et al., (2018) review a sample of over 200 published articles to examine how multidisciplinary curricula were developed through sustainability integration around the world. They found that in most instances there was little agreement and lack of clarity regarding the multidisciplinary aspect with focus often diverted to assessing the extent to which sustainability was embedded in the curriculum. Most studies reported on diversity of values held between students and academics regarding sustainability. Students for instance were found to prioritize environmental aspects of sustainability whilst academics focused on social and economic issues. In addition, there were differences in how sustainability content was integrated in the courses, ranging from revisions made to existing modules to designing entirely new programmes.

In addition to sustainability, some scholars suggest that project-based teaching offers opportunities for multidisciplinary learning. Research that reflects upon experiences of designing and developing multidisciplinary curricula through project-based work in engineering mostly highlights the benefits of such an approach, with little or no critique. Burnell et al. (2003) suggest that students view learning through multidisciplinary projects to be more challenging but also more rewarding. Heikkinen and Isomöttönen (2015) similarly report on students positive experience of undertaking a multidisciplinary course at the University of Jyvaskylä in Finland. They suggest that working in a multidisciplinary course enabled most students to better understand their own discipline giving them a clearer sense of professional identity, as well a better understanding of multidisciplinarity. Drawing on qualitative interviews as well as content analysis of students' project reports, they conclude that students view multidisciplinary approaches as beneficial and often develop a clearer understanding of their own disciplines. They also reflect upon the need for a supportive educational team environment as some students found the experience difficult. Bhandari et al. (2011) discuss the implications of introducing project based multidisciplinary learning in an existing sustainable engineering course. By conducting a survey with students as well as analysing the project reports, they suggest that $72 \%$ of the students agreed that their ability to consider multiple disciplinary views and constraints improved as a result of the project-based curriculum integration. An improved understanding of sustainability was also noted in the review of the project reports. Hotaling et al. (2012) compare a multidisciplinary and mono-disciplinary version of a capstone engineering course developed across two different faculties. 
They suggest that students who undertook a multidisciplinary version of the course had a better employment outcomes and feedback from industry partners than students from mono-disciplinary versions of the course.

A new generation of multidisciplinary engineering programmes has been driving engineering educational reform in several institutions drawing on innovative teaching methods including active project-based learning (Graham 2018). Graham (2018) identifies that these innovative curricula are often shaped by specific regional needs and constraints, and their success relies on them being embedded in a faculty culture of educational innovation and the use of new tools to support educational exploration and student assessment. The report identified a number of "emerging leaders" including UCL Faculty of Engineering Sciences (UK), TU Delft (Netherlands) and Charles Sturt University (Australia). Multidisciplinary educational practices that were highlighted at these institutions included "usercentered design, technology-driven entrepreneurship, active project-based learning and a focus on rigour in the engineering 'fundamentals"'. The "emerging leaders' were seen to move towards "socially relevant and outward-facing engineering curricula...", which "emphasize student choice, multidisciplinary learning and societal impact, coupled with a breadth of student experience outside the classroom". These experiences were identified as being delivered "with an integrated and unified educational approach... designed from a blank slate or ... the result of a recent systemic reform".

Project based-learning is also discussed through integrating design-studio or makerspaces within engineering curricula, both as innovative physical entities as well as sociocultural shifts in the learning process. Wilczynski (2015) review a number of recently developed engineering programmes in the USA that have developed or integrated design studio/maker spaces within their courses. The review illustrates the diversity of methods for incorporating design studio/maker spaces in engineering curricula, with some key overlapping processes. Key practices that are found in all course developments are suggested to be shaped by organizational, social as well as academic processes including: a shared academic mission, appropriate resourcing and multi-disciplinary support, open environments and spatial flexibility enabling multiple uses.

Forest et al., (2014) describe through one of the cases reviewed above, the process of developing a design studio environment termed an 'Invention Studio' as part of all engineering curricula at Georgia Institute of Technology. They reflect upon the multidisciplinary benefits of such an approach through conducting a large survey with 1000 students, suggesting that the underlying motivation, organization, facilities, outreach, safety and funding conditions are critical to its success. Though not focusing on the process itself, Rangel et al., (2016) explore student experiences of implementing design studio learning at the Faculty of Engineering at the University of Porto. They found that students' ability to acquire knowledge' and motivation to succeed were improved as a result.

Whilst not referring to design studios as a spatio-social approach to educational integration, Telenko et al., (2014) reflect upon the use of designettes in engineering curricula as a multidisciplinary learning activity. They argue that designettes enable the development of a 'culture of design', exposing the challenges that come with design. Drawing on surveys with over 300 students, designettes were found to increase 'students' awareness of applications and learning of content' as well as 'increase students' self-perceptions of their ability to solve multidisciplinary problems'.

In addition to sustainability, design studio and project-based learning, Becerik-Gerber et al., (2011) suggest BIM as a way of achieving a multi-disciplinary environment. BecerikGerber et al., (2011) conducted a survey across architecture and engineering programmes in the USA with the aim of understanding approaches to achieving multidisciplinary 
curricula. The survey measured the extent to which educators perceived multidisciplinary integration to be BIM or sustainability driven. Drawing on 121 respondents, they report that $56 \%$ of engineering programmes view BIM as a way of developing multidisciplinary learning.

Whilst above discussed studies offer helpful insights into ways multidisciplinary learning has been approached as well as their effects on student experience, the primary focus is on integrating multidisciplinary learning or in some instances architectural practices in engineering curricula, evidencing a lack of studies that consider the process of embedding engineering approaches in architecture courses. Exceptions include a study by Oliveira and Marco (2017) that reflects upon the effects of transdisciplinary learning in a combined fully accredited (RIBA/CIBSE) architecture and environmental engineering course in the South West of England. Their study, however, is largely focused on the effects of multidisciplinary learning, rather than on the development of it. Similarly, Olsen and MacNamera (2014) focus on the potential effects of architecture and engineering educational collaborations as providing and promoting workplace success for both disciplinary cohorts. In addition, MacNamera's (2011) paper, considering implications of an attempt to develop an integrated course, argues that in their formative education, engineering and architecture students have few opportunities for multidisciplinary learning. Despite highlighting the importance of multidisciplinary learning, MacNamera (2011) and Olsen and MacNamera (2014) reflect upon previously documented studies concluding that engineering pedagogy has more to gain from architecture than vice-versa.

Mistur (2011) reflects on the obstacles of integrating engineering content into architectural curricula and on the role of collaboration in realising progressive design solutions. Mistur (2011) describes a tendency within architectural education to prioritise traditional or conventional design knowledge over critical or collaborative learning, suggesting that resistance to facilitate multidisciplinary learning may lead students to develop siloed experiences.

This section has identified that there is scarce research into the development process of integrating engineering content into architectural courses. Furthermore, through reviewing existing studies it is evident that few reflect upon the process of designing a multidisciplinary curriculum or course in general, in the disciplines of engineering and built environment. The following sections discuss the processes academics and others undertook to create and enable a multidisciplinary course in engineering education.

\section{Reflecting upon multidisciplinary engineering curricula design: insights and learning}

Research that reflects upon experiences of designing and developing multidisciplinary curricula in engineering mostly highlight lessons learnt, student views and recommendations for the future. Few studies describe the process of designing and evaluating a multidisciplinary curriculum from institutional or educators' perspectives. A few notable exceptions include works by Graham (2018), Clevenger et al., (2017) and Richter and Paretti (2009) as discussed below.

Graham (2012), in a report written on behalf of Royal Academy of Engineering and MIT, discusses several case studies in Engineering departments around the world, making a set of recommendations for departments and schools wishing to embark on systemic change, based on a consultation with 70 academics and leaders in engineering, 
and on interviews with 53 people involved in the case studies. One of the case studies was identified at UCL, of a revised curriculum in Civil and Environmental Engineering, a broad department-wide change in curriculum which was achieved in a "mouldbreaking and rapid" manner. One of the key ingredients in this curriculum were the integration of studio style teaching on integrated design projects following an architectural educational model. It was crucial that this curriculum received accreditation in Civil Engineering, despite being seen initially as based on some controversial change. The report identifies that the vision for the curriculum "... was also seen to be addressing a number of fundamental concerns about UK engineering education, such as how to widen participation in engineering, particularly amongst girls, and how to improve the leadership position of UK engineers in an increasingly globalised industry." In discussing the process of introducing curricular change, the report found evidence that a combination of strong departmental leadership and widespread faculty engagement in a process of both bottom-up and top-down educational reform, which were informed, coherent and ambitious, led to a successful process and produced a pioneering, interdisciplinary undergraduate curriculum.

Graham (2018) evaluates an international portfolio of multidisciplinary general engineering curricula, identifying key challenges to successful curriculum development as being primarily spatial and institutional. Lack of flexibility of university learning spaces was viewed by most participants across multiple studies to be a significant challenge in developing multidisciplinary curricula. In many instances educators reported having to "configure the projects to fit the spaces we have, rather than the other way around." Adding to already loaded existing curricular content was also found to be a significant challenge across many institutions, with some finding it difficult to reduce existing content.

Clevenger et al. (2017) evaluate the curriculum outcomes of a multidisciplinary course by focusing on student and industry feedback. Their findings suggest that academic planning of multidisciplinary curricula should involve greater programmatic and course level coordination as well as opportunities for iteration. Richter and Paretti (2009) also identify key challenges to successful delivery of multidisciplinary courses as being faculty driven. The findings suggest that students (1) lack the ability to connect interdisciplinary subjects to their own more narrowly defined fields of expertise, and (2) fail to identify and value the contributions of multiple fields to complex problems. This paper concludes with suggested teaching interventions to address these barriers including facilitation of greater dialogue on modes of thinking across disciplines.

Wolff and Luckett (2013) study the underlying conceptual basis for multidisciplinary engineering curricula through 'contextual and conceptual' elements drawing on theoretical tools developed by Bernstein (2000) and Maton (2009). Drawing on a sample of four students in a cohort of 20, they study how students approach problem solving with a view to understanding their knowledge integration processes. The findings suggest that multidisciplinary learning is found to be shaped by a more complex knowledge structure requiring a complex praxis capability: 'the ability to appropriately access relevant theory from the core disciplines (the 'know-why') as well as procedural 'know-how', and to integrate these in a particular context of application'.

An early study by Miller and Olds (1994) offers insights into the process of developing a multidisciplinary engineering curriculum integrating eight academic disciplines. The process is described as containing several sequential stages including survey with industry firms, developing management structures to coordinate various departments and designing the course structure. Outcomes of the developed course were continually assessed in terms 
of student and industry firm perceptions enabling the course and management structures to be enhanced.

All the studies discussed above, develop multidisciplinary content mostly within general or civil engineering courses. We are unaware of any accounts to date that discuss integration of three building design professions or the development of an entirely new discipline.

\section{Research methods}

The starting point for the research approach was an interest in exploring the conditions and approaches to developing multidisciplinary content for the UCL MEng Engineering and Architectural Design course. The methodological approach was developed by combining several qualitative strategies: interviews and documentary analysis (Mason 2017). Multiple methods including interviews, observations or documentary data can be used to address particular research questions to explore different parts of a process or to address different analytical levels (Patton 2002).

The initial part of the research approach explored how understandings on the multidisciplinary content and delivery mechanisms developed during the initial stages in course development. Documentary evidence was collected including minutes of meetings held between key course developers as well as course module presentations and course descriptions. Having gained an understanding of how initial understandings on multidisciplinary course content may have developed over time, the second part of the research examined educators' and industry advisors' views on the curriculum design process as well as the multidisciplinary content of the course. 13 participants including educators and industry advisors involved in developing the course were contacted initially, with 7 interviews held over phone and skype (see Table 1 for indication of data types collected). As the interviews were applied in order to add depth to the study and explain and explore specific issues regarding multidisciplinary content and mechanisms for delivery in the course, a semistructured approach was used in the study.

The interview protocol was structured into three main sections. As such, the protocol has a sequence of themes as well as suggested questions and at the same time an openness to sequence and form of questions (Kvale 1994). The first section was introductory and related to exploring participants' role and interests in developing the course. These introductory questions were designed to set the scene and provide an insight into the participants' role in the development process. The second set of questions explored participants' expectations of the course as well as future role graduates may play in the built environment. The third set of questions focused on exploring attitudes towards developing multidisciplinary course content. Each interview concluded with asking whether participants had additional thoughts or information they thought relevant which had not been discussed. All interviews were tape-recorded and transcribed and lasted between 30 and $45 \mathrm{~min}$. The interview was recorded verbatim avoiding the issues of poorly recorded data. Given the confidential nature of the evidence, it was agreed with every participant that they would remain anonymous. At the end of the interview time was spent collating thoughts on some of the highlights of the interviews and observations not captured through recording.

Once all the data had been collected separately, the analysis focused on rereading the archival and interview data to gain an understanding of all the key issues and developments. These materials constituted an initial 'data base' (Mason 2017). The data base was then analysed once again highlighting 'text segments' that discussed conditions and 


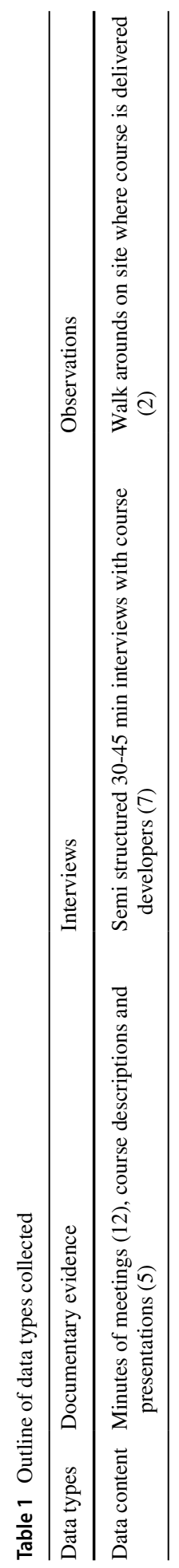


delivery mechanisms for multidisciplinary education. Codes were then developed to represent identified themes. A theme captures something important about the data in relation to the research question and represents some level of patterned reasoning within the data set (Richards and Morse 2012). Descriptive codes are valuable in getting the analysis started as well as summarizing segments of the data. An analytic code pulls together material into smaller higher-level units.

Coding was carried out in two stages once all the data collected and analysed separately had been organized into a data base. The initial stage of analysis included rereading the documentary evidence including minutes of initial development meetings, module descriptions as well as analysis of previous external examiners reports undertaken by course academics. The initial analysis of external examiners reports undertaken by academics developing the course was collated into the initial course documentation. This demonstrated an initial programme design against EC AHEM and ARB learning outcome criteria. The second stage of the analysis focused mainly on including preliminary thematic coding of documentary evidence and the interview data identifying some descriptive codes relating to developing multidisciplinary courses: (1) expectations for the course; (2) attitudes towards its development; (3) conditions for development. These are discussed in further detail in following sections.

\section{Empirical context: the MEng engineering and architectural Design course}

The course is designed to address the expectations of three accreditation bodies, ARB, JBM and CIBSE, and thus to meet both Engineering Council and Architecture degreelevel requirements. These requirements provided the broad boundaries within the curriculum design team operated. Key competencies and skills were identified, and the curriculum follows areas where there was significant overlap in required content, skills and competencies developed by the students. The course draws on expertise from The Bartlett School of Architecture, the UCL Institute for Environmental Design and Engineering and the Department of Civil, Environmental and Geomatic Engineering as well as leading practitioners from across the globe.

The course learning is embedded within the London Olympic Park site Here-East equipped with a state-of-the-art fabrication resource including structural, material and

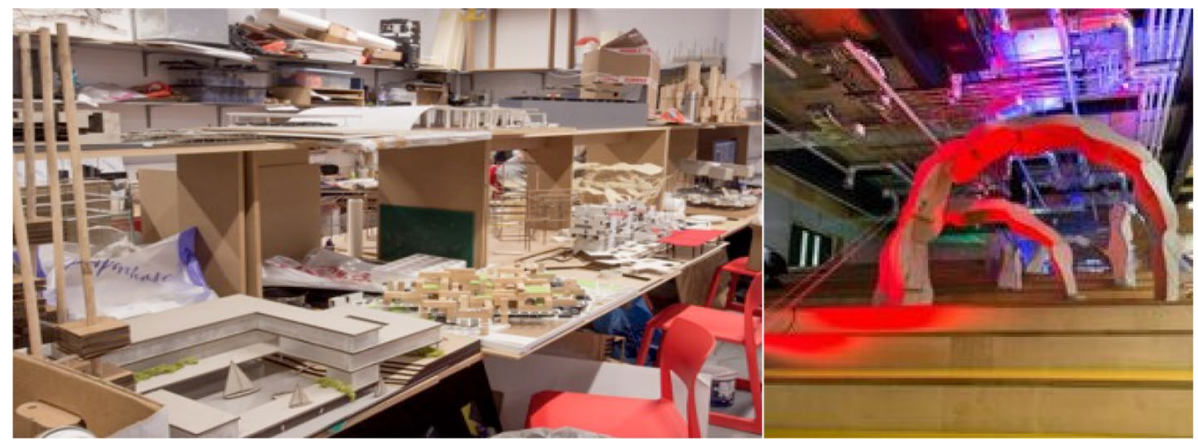

Fig. 1 Illustration of Here East fabrication and making spaces 
human environmental chambers, robotics, $3 \mathrm{~d}$ printing, $\mathrm{CNC}$ milling and water jet cutting alongside woodworking and metalworking workshops (see Fig. 1).

Here East houses a sequence of multi-functional and adaptable large-scale spaces from (1) public/exhibition/foyer/studios, through to (2) large volume collaboration hub for demonstrations/assemblies and gatherings of variable scale, through to (3) large volume fabrication hub for large scale manufacture and assembly, through to (4) large volume research hub for dedicated projects at an advanced level, with environmental chambers. Students are provided with an entirely novel environment that is part gallery and archive, part auditorium and theatre, part studio and office, part laboratory and factory, and part social generator, all in one envelope.

The course structure (see Fig. 2 and Table 2) is designed around this constellation and thus provides a novel and provocative set of resources through which a new generation of pioneering graduates are able to develop.

Student experience and teaching delivery is centred on a combination of the design studio model that underpins the Bartlett Schools of Architecture's ARB/RIBA validated programmes, CEGE's experience in developing JBM-accredited programmes centered on problem-based learning, as well as IEDE's research-based learning experience based on joint education of architects and engineers for over 40 years. This unique mix, placing creativity and design at the centre of a multidisciplinary built environment education, challenges traditional models of built environment educational models. It allows multidisciplinary modes of integrated assessment, whereby focus is placed on developing interdisciplinary vocabularies and problem based learning. The course structure also allows students the opportunity to understand and develop advanced design methodologies whilst

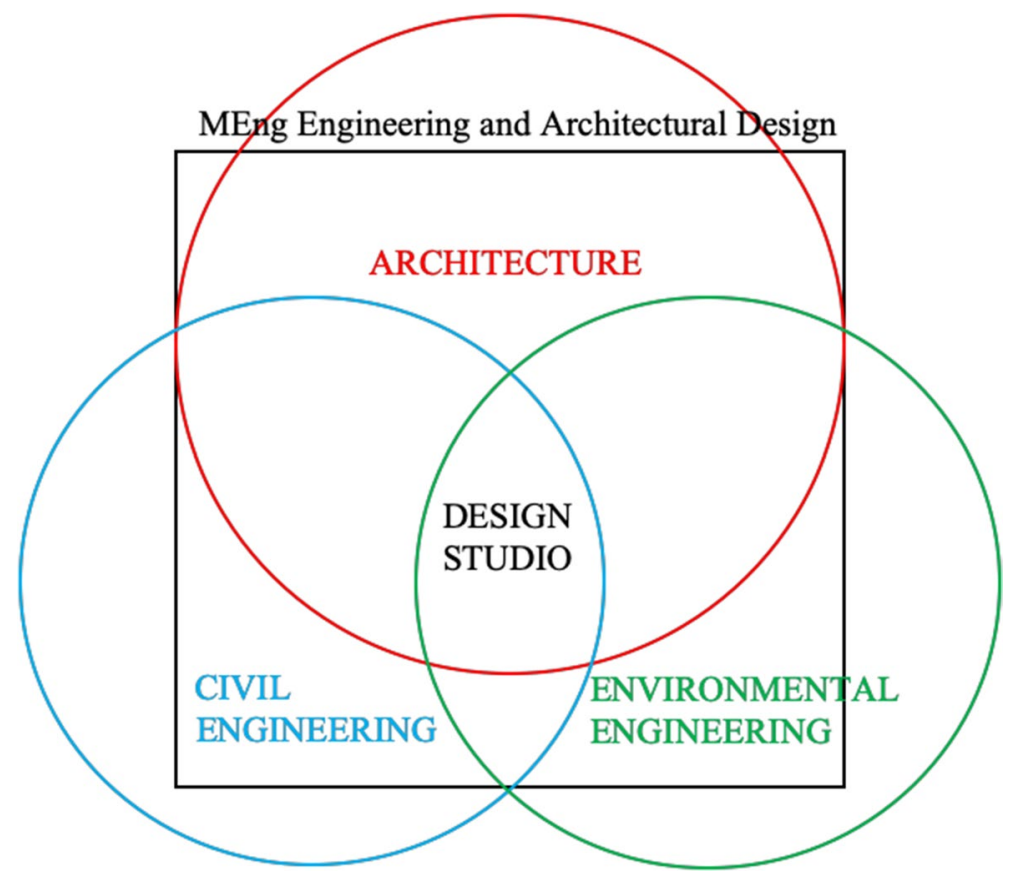

Fig. 2 Course structure diagram 


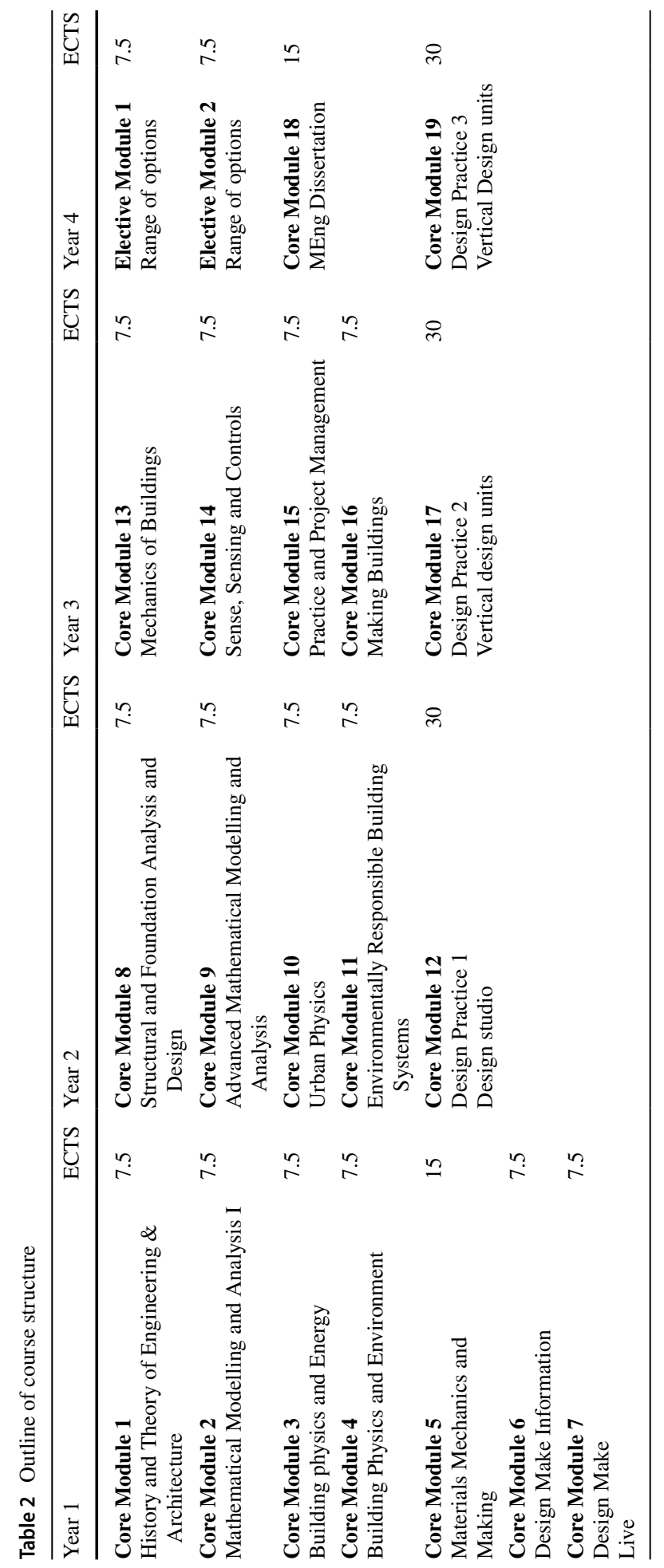


acquiring expertise on how they are augmented and resolved through engineering knowledge and research rigour (see Table 2).

\section{Findings}

The findings suggest that multidisciplinary content is shaped by shared approaches to personal expectations and professional attitudes towards the course as well as a sense of uniqueness and a wider willingness to accept risks. Regarding personal expectations two themes emerged including a focus on positive outcomes and shared starting points. Referring to professional attitudes, discussions focused on maintaining the wider vision (see also Table 3).

\section{Personal expectations: Shared starting points and positive outcomes}

When discussing their experience of developing the multidisciplinary course content that seeks to bridge architecture, structural and building services engineering learning outcomes, participants largely drew on their personal experience of working or learning in an interdisciplinary setting. This personal experience of working closely with other disciplines was described as invaluable to the course development discussion process. One of the participants for instance discusses their early experience in engineering practice as 'an early taste of success(full) interdisciplinary working' (Participant 1). Another participant discusses how acknowledgement and understanding of the course leaders' disciplinary standpoints was a critical component to enabling initial discussions:

...The first thing that helps with ...the 3 disciplines are very well established already...if these were new disciplines that would be a complicated challenge... what we're trying to do is connect silos...the first thing is to try to understand the rigour of each discipline... (Participant 3)

Some participants conveyed a sense of a 'common desire' to achieve an 'integrated approach' that 'realised the importance of each team'. In addition to shared starting points most participants conveyed a sense of having 'a blanc sheet of paper' when developing the course content which set apart the course from other similar interdisciplinary integrated courses that had to share content or modules with other courses. The ability to truly develop appropriate content that set out the vision of the course was found to be a critical component of the discussions' successful outcome.

We really started with a blank sheet of paper...that is a difference with many other integrated courses...many others use existing modules and stich them together...that wasn't a driving force..we actually started what we were delivering in each year and then determining module content that would support that... (Participant 2)

Overall, participants conveyed a sense of a positive outcome driven by a common understanding and desire to successfully integrate diverse disciplinary perspectives. One participant conveys the shared values as being equally important to all and based on egalitarian principles as a key course enabler: 


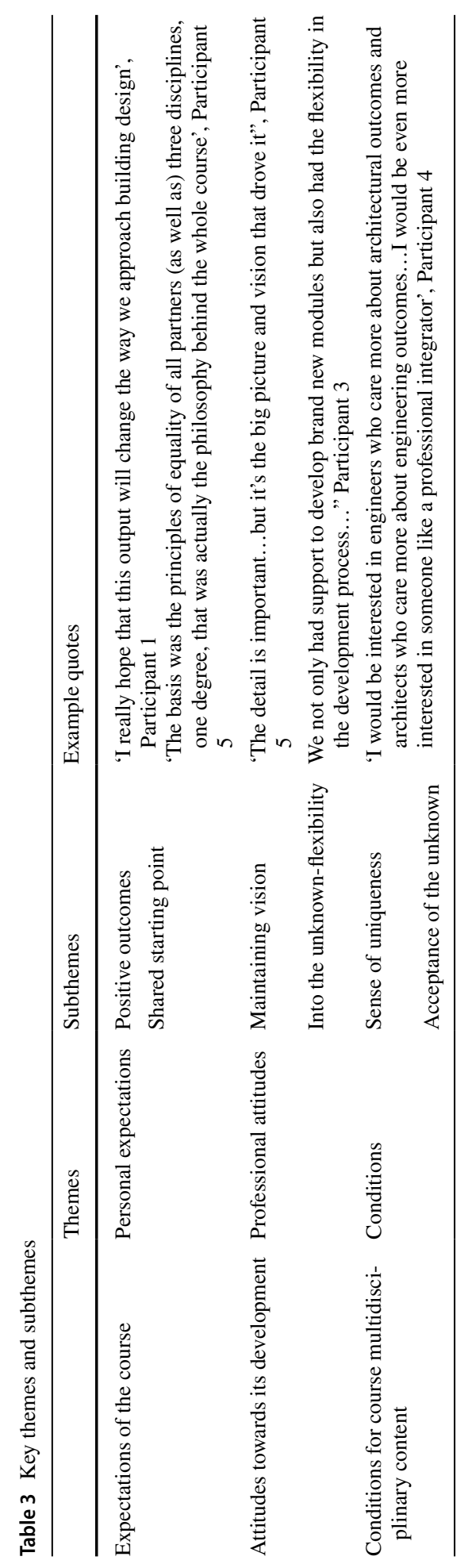


...the (basis) was the principals of equality of all partners and the basis of three disciplines, one degree, that was actually the philosophy behind the whole course... (Participant 5)

Many participants discussed the opportunities the course may offer to help develop future industry leaders that could easily and with skill bridge disciplinary boundaries, having developed the ability to 'speak many languages'. Whilst many described the future graduate to be a new type of professional, a 'building designer' as well as a 'specialist generalist', many also discussed the potential other possibilities the course could offer to a developing industry need for greater collaboration and integration.

\section{Professional attitudes: into the unknown/maintaining vision}

Participants discussed the process of developing the course as being challenging as well as open and a venture into the unknown. Many participants stressed the importance of the course being a new type of discipline- neither engineering nor architecture. One of the participants suggested the course needed the freedom and flexibility to continue to develop:

...It's not an engineering course...it's a new hybrid discipline...it is possible it may have a new name in the future..that will only come into play when we understand how unique it is to be operating in proximity of its own trinity.... (Participant 2)

Many recognised the need to be non-prescriptive about the course description to an extent and enable the course the evolve and develop. For one participant the course developed as an enmeshing of two disciplines:

...I would be interested in engineers who care more about architectural outcomes and architects who care more about engineering outcomes...I would be even more interested in someone like a professional integrator... (Participant 4)

In addition to viewing the process of developing the course as a venture into the unknown, many participants reflected upon the need for institutional support as a prerequisite for the course success:

...We not only had support to develop brand new modules but also had the flexibility in the development process... (Participant 3)

I had a very understanding a good head of department at that time, who actually understood the value of this kind of education and he thought that is great and I had the support of colleagues ( Participant 5)

Institutional support as well as having the possibility of the course being delivered in a purpose-built facility driven by a design studio style teaching delivery were important factors in maintaining vision as well as overcoming cross departmental challenges. Many participants discussed the importance of 'maintaining ambitious vision' as an important aspect of the course development conversations.

...The thing that kept it going is to aim for the higher hanging fruit....and maintain ambitious vison at the centre of all conversation...were all very aware that we can get bogged down in detail...those barriers can be removed if you want them to be removed...there was huge determination to do the right thing..to put problems and 
obstacles to one side... were looking for people and programme to get around obstacles... (Participant 7)

Whilst most participants recognised the need to resolve detail and talk through divergent objectives, many reflected upon the need for 'the big picture and vision'. Negotiating divergent aspirations driven by three departments coming together was often brought up in discussions. One of the participants (Participant 1) discussed their role of negotiator as a:

...A Kissinger type activity..where we had the various fractions..who wanted the course to be $90 \%$ their current BEng...and our objective..is melded the different disciplines and providing some common understanding of areas that could be covered that delivered learning objectives that suited a number of areas....

Some participants described the importance of recognising 'different personalities', working across shared ideals in order to deliver 'a good deal'. In order to negotiate though, it was recognised that all participants came to the discussions with a shared 'common goal.'

\section{Conditions: identifying uniqueness/acceptance of the unknown}

In addition to personal expectations and professional attitudes many participants recognised the importance of certain facilitating conditions. In addition to institutional support, purpose-built facilities as well as resources, most participants conveyed the course through a 'sense of uniqueness'. Uniqueness was discussed in terms of the course content, its focus on design studio style teaching as well as the uniquely designed spaces within which it is delivered.

One of the participants describes the course 'uniqueness' as a different form learning 'that gives intimate proximity to other close sibling disciplines'. For many uniqueness was identified through the different modes of learning the course provided.

...Were interested in this programme being quite unique and different...studio based learning is at its centre... and it's something the other two disciplines are not familiar with..likewise..in the other direction...the rigour of research design space going on are not happening in design programmes anymore and they should be... (Participant 5)

Further to identifying a sense of uniqueness, one of the participants reflected upon the need to 'not know' what kind of graduate the course may enable. The participant described the traditional ways of working and valuing skills of a specialist rather than a generalist. The participant then discussed the need to shift from the need for a specialist to a need for an individual that integrates specialisms:

...The traditional view is you have specialists and generalists... and generally we don't like generalists because they are not as good as specialists...and what we've got to try and develop is this understanding that the integration of specialists is a specialism in itself...

The need for graduates who also combined non-traditional engineering roles with skills in 'integration, hunch, intuition, communication and empathy' as well as graduates who could be 'wider thinkers' was often reflected upon. One participant illustrates this by illustrating how module content was developed to enable this multidisciplinary ethos: 
...Understanding actually how to deliver that, so our option at that stage, we were looking at how we wanted to create modules for this course and we decided that having, for example, thermal properties of the materials as part of one module and then construction properties of materials as the other module would be a completely bolt on approach and so we actually rejected that, so multidisciplinarity, for example, has been developed for this course, doesn't have traditional, fundamental modules such as thermal dynamics or such as fluid mechanics, that would be expected from a civil engineering course or a building services engineering course... Participant 5

Overall participants who took part in developing the course content, discussed the course as meeting a need that is not fully articulated yet but emerging. The discussions of the course development mostly focused on developing a future graduate, one that is conversant in architecture, building services as well as structural engineering as well as one that offers a hybrid of all. Most participants discussed the need for openness to compromise on learning outcomes across and between disciplinary boundaries, to take advantage of the institutional support, the new facilities, the industry need and most of all ability to design bespoke modules. Participant 5 reflects upon the potential for the future graduate to change the industry:

I would like this course to be seen as training creative and highly technically, literate building designers. People actually who can generate some change in industry and hopefully for the better and again, the minimum expectations will be that no matter if they undertake these kind of traditional jobs in traditional, fragmented building design industry, then they would understand the language of other three disciplines, so they would be empowered by having intimate knowledge of processes, including engineering and architectural design, so they could provide much more robust discussion and maybe provide more integrated solutions than other people could... Participant 5

Whilst some participants were industry representatives, and other academics, the findings suggest a shared vision and willingness to explore the unknown. It is still too early to fully understand the way the course is evolving as well as the effects of merging disciplinary thinking within one curriculum.

\section{Discussion and conclusion}

Research in engineering and architecture education has maintained the need for developing integrated multidisciplinary content as an imperative for equipping future built environment design professionals (Graham 2018). The need for interdisciplinary, collaborative and integrated working is especially pronounced in the built environment (Becerik-Gerber et al. 2011; Morrell 2015). However, despite the calls, few studies have discussed the approaches to enabling and developing multidisciplinary content. Whilst, literature discussed in the above section suggests multidisciplinary content is provided through additional projectbased or sustainability learning (Thürer et al. 2018) as well as technology such as BIM (Becerik-Gerber et al. 2011), the outcomes of such a development as well as the mechanisms that lead to it are poorly understood. Also, a number of studies suggest conditions to enable multidisciplinary content are largely institutionally or faculty driven (Richter and Paretti 2009). 
Findings in this study suggest personal and professional expectations and attitudes of those involved in developing the course content are critical. The study shows that multidisciplinary content for the new MEng course was driven by a shared vision, an openness and ability to delve into the unknown. Whilst many participants recognised the importance of institutional support as well as appropriate facilities and resources as the literature above suggests, these conditions were not the only mechanisms that mattered. In addition to conditions, it was the people, their mutually reinforcing disciplinary perspectives and shared vision that had the greatest role to play. Some participants described their personal takes and experiences of the process as negotiators, others as visionaries; for many the detail was critical but overall there was an acceptance into the unknown, a flexibility of thought and purpose and above all a common goal. The findings support and extend observations in Clevenger et al. (2017) study on the importance of a shared programmatic and course level vision as well as providing opportunities for iteration and risk taking in facilitating multidisciplinary curricula. Participants conveyed the importance of fostering a shared vision regardless of disciplinary experiences or differences in order to develop a collective multidisciplinary approach. They also conveyed the potential such an environment within which different disciplinary positions can be shared as a source for future growth in developing new built environment roles.

Whilst the implications of this study are primarily in developing engineering and architecture curricula, there are helpful insights that might benefit STEM education more widely. For instance, the importance placed on personal experiences and expectations. Most participants engaged in developing this course had some prior experience of multidisciplinary curricula, either as students or educators in past institutions. There may be further helpful benefit in developing STEM professional courses or seminars that could offer insight and experience of learning in a multidisciplinary environment, leading to a positively led curricular approach that merges and draws on different disciplines.

Whilst the participants did not reflect on the role of the environment both on terms of equipment or space needs, it may have been helpful to further explore how the physical design of a very novel and bespoke space facilitated or enabled certain curricular understandings or discussions. Future studies could further explore how this novel environment may inform or enable particular types of activities and learning environment that otherwise may not have been possible. It is also recognised that this study is based on one course. Future studies could compare course development processes with regards to multidisciplinary content of two or more courses, perhaps across different sectors or between different countries. Future studies are also needed to analyse further the role different people and their activities, vision and expectations play in developing multidisciplinary content.

Open Access This article is licensed under a Creative Commons Attribution 4.0 International License, which permits use, sharing, adaptation, distribution and reproduction in any medium or format, as long as you give appropriate credit to the original author(s) and the source, provide a link to the Creative Commons licence, and indicate if changes were made. The images or other third party material in this article are included in the article's Creative Commons licence, unless indicated otherwise in a credit line to the material. If material is not included in the article's Creative Commons licence and your intended use is not permitted by statutory regulation or exceeds the permitted use, you will need to obtain permission directly from the copyright holder. To view a copy of this licence, visit http://creativecommons.org/licenses/by/4.0/.

\section{References}

Becerik-Gerber, B., Gerber, D. J., \& Ku, K. (2011). The pace of technological innovation in architecture, engineering, and construction education: integrating recent trends into the curricula. Journal of Information Technology in Construction (ITcon), 16(24), 411-432. 
Bhandari, A., Ong, S. K., \& Steward, B. L. (2011). Student learning in a multidisciplinary sustainable engineering course. Journal of Professional Issues in Engineering Education and Practice, 137(2), 86-93.

Bernstein, B. B. (2000). Pedagogy, symbolic control, and identity: Theory, research, critique (Vol. 5). Lanham: Rowman \& Littlefield.

Borrego, M., \& Newswander, L. K. (2008). Characteristics of successful cross-disciplinary engineering education collaborations. Journal of Engineering Education, 97(2), 123-134.

Burnell, L. J., Priest, J. W., \& Durrett, J. R. (2003). Assessment of a resource limited process for multidisciplinary projects. ACM SIGCSE Bulletin, 35(4), 68-71.

Clevenger, C. M., Brothers, H., Abdallah, M., \& Wolf, K. (2017). Initial assessment of a newly launched interdisciplinary construction engineering management graduate program. Journal of Professional Issues in Engineering Education and Practice, 143(3), 04017001.

Dym, C. L., Agogino, A. M., Eris, O., Frey, D. D., \& Leifer, L. J. (2005). Engineering design thinking, teaching, and learning. Journal of Engineering Education, 94(1), 103-120.

Forest, C. R., Moore, R. A., Jariwala, A. S., Fasse, B. B., Linsey, J., Newstetter, W., \& Quintero, C. (2014). The Invention Studio: A University Maker Space and Culture. Advances in Engineering Education, 4(2), n2.

Graham R (2012) Achieving excellence in engineering education: the ingredients of successful change. Massachusetts Institute of Technology

Graham R (2018) The global state of the art in engineering education. Massachusetts Institute of Technology

Heikkinen, J., \& Isomöttönen, V. (2015). Learning mechanisms in multidisciplinary teamwork with real customers and open-ended problems. European Journal of Engineering Education, 40(6), 653-670.

Hotaling, N., Fasse, B. B., Bost, L. F., Hermann, C. D., \& Forest, C. R. (2012). A quantitative analysis of the effects of a multidisciplinary engineering capstone design course. Journal of Engineering Education, 101(4), 630-656.

Jones, A., \& Gloster, D. (2018). Education futures - a report by Alan Jones and David Gloster to the Board of The Royal Institute of British Architects (approved 13 Sept 2018) and endorsed by the Council of the Royal Institute of British Architects (27 Sept 2018): Developing education to drive progress, modernity, and inclusivity in the profession. Royal Institute of British Architects.

Kvale, S. (1994). Interviews: An introduction to qualitative research interviewing. Beverley Hills: Sage Publications, Inc.

Macdonald, J. A. \& Granroth, M. (2013). Multidisciplinary AEC education utilising BIM/PLIM tools and processes. In IFIP international conference on product lifecycle management (pp. 663-674). Berlin: Springer.

MacLaren, A., Wilson, M., Simmonds, R., Hamilton-Pryde, A., McCathy, J., \& Milligan, A. (2017). Educating Students for the Collaborative Workplace: Facilitating Interdisciplinary Learning in Construction Courses. International Journal of Construction Education and Research, 13(3), 180-202.

MacNamera, S. (2011). Trans-disciplinary design teaching for civil engineers and architects, lessons learnt and future plans. Washington: American Society for Engineering Education.

Mason, J. (2017). Qualitative researching. Beverley Hills: Sage.

Maton, K. (2009). Cumulative and segmented learning: exploring the role of curriculum structures in knowledge-building. British Journal of Sociology of Education, 30(1), 43-57.

Mistur, M. (2011). Design leadership: three pedagogical pairings for performative. Rensselaer Polytechnic Institute.

Miller, R. L., \& Olds, B. M. (1994). A model curriculum for a capstone course in multidisciplinary engineering design. Journal of Engineering Education, 83(4), 311-316.

Morrell, P. (2015). Collaboration for change: The edge commission report on the future of professionalism 1 edge debate. London: The Edge Commission.

Morse, W. C., Nielsen-Pincus, M., Force, J. E., \& Wulfhorst, J. D. (2007). Bridges and barriers to developing and conducting interdisciplinary graduate-student team research. Ecology and Society, 12(2).

National Academy of Engineering (2013). Educating engineers: Preparing 21st century leaders in the context of new modes of learning, USA

Oliveira, S., \& Marco, E. (2017). Preventing or inventing? Understanding the effects of non-prescriptive design briefs. International Journal of Technology and Design Education, 27(4), 549-561.

Olsen, C., \& MacNamera, S. (2014). Architecture and engineering pedagogy. In Collaborations in architecture and engineering (pp. 182-199).

Patton, M. Q. (2002). Two decades of developments in qualitative inquiry: A personal, experiential perspective. Qualitative Social Work, 1(3), 261-283.

Rangel, B., Sá, A. V., Guimarães, A. S., \& Alves, F. B. (2016). Integrated design concept in civil engineering education. International Journal of Engineering Education, 32(3(A)), 1279-1288.

Richter, D. M., \& Paretti, M. C. (2009). Identifying barriers to and outcomes of interdisciplinarity in the engineering classroom. European Journal of Engineering Education, 34(1), 29-45. 
Richards, L., \& Morse, J. M. (2012). Readme first for a user's guide to qualitative methods. Beverley Hills: Sage.

Sunthonkanokpong, W. (2011). Future global visions of engineering education. In Proceedia engineering (pp. 160-164).

Telenko, C., Camburn, B., Hölttä-Otto, K., Wood, K., \& Otto, K. (2014). Designettes: New approaches to multidisciplinary engineering design education. In ASME 2014 international design engineering technical conferences and computers and information in engineering conference (pp. V003T04A003-V003T04A003). American Society of Mechanical Engineers.

Thürer, M., Tomašević, I., Stevenson, M., Qu, T., \& Huisingh, D. (2018). A systematic review of the literature on integrating sustainability into engineering curricula. Journal of Cleaner Production, 181, 608-617.

Wilczynski, V. (2015). Academic maker spaces and engineering design. American Society for Engineering Education, 26, 1.

Wolff, K., \& Luckett, K. (2013). Integrating multidisciplinary engineering knowledge. Teaching in Higher Education, 18(1), 78-92.

Publisher's Note Springer Nature remains neutral with regard to jurisdictional claims in published maps and institutional affiliations. 